\title{
Kinderfernsehen bei sozial benachteiligten Kindern
}

Ingrid Paus-Hasebrink, Michelle Bichler und Christine W. Wijnen

\section{Hohe gesellschaftliche Verantwortung - grosse Forschungsdefizite}

Ist von Kindern und Medien - dies gilt auch für das Kinderfernsehen - die Rede, so dreht sich die nur selten sachlich und oft eindimensional, mit Blick vor allem auf die Medien, geführte Debatte zumeist um die Dauerbrenner "Gewalt und Werbung». Das Thema Medien bzw. Fernsehen und Kinder aber erfordert den Blick auf zumindest zwei Ebenen: die Ebene der Produktion und des Angebots sowie die Ebene der Rezeption (vgl. Paus-Hasebrink/Woelke/Bichler/Pluschkowitz 2006). Beide Ebenen sind zudem nur im Kontext gesellschaftlicher Wandlungsprozesse angemessen zu verstehen und zu beurteilen. Dies bedeutet etwa im Hinblick auf die Frage nach dem Umgang von Kindern mit Medienangeboten, dass zumindest zwei grundlegende Aspekte mit berücksichtigt werden müssen: die Veränderungen der Medienumwelt (Vervielfältigung und Ausdifferenzierung von Medienangeboten) und der Wandel der familialen Lebensformen. Rund um die Thematik 'Kinder, Kindheit und Medien) ist jedoch vor allem einem Punkt bislang in der öffentlichen Debatte und auch in der Forschung noch vergleichsweise wenig Beachtung geschenkt worden: der Milieuzugehörigkeit der Kinder als Einflussfaktor in Bezug auf ihren Umgang mit Medien und den daraus resultierenden Auswirkungen auf ihre Sozialisation - und dies speziell in sozial schwächeren und anregungsärmeren Milieus. ${ }^{1}$ Denn wie Kinder Medien gebrauchen und welchen Stellenwert sie Medien zuschreiben, hängt unter anderem auch von den soziokulturellen Voraussetzungen in ihrer Lebenswelt ab. Hier sind vor allem die Faktoren Schichtzugehörigkeit, Bildungsniveau, Familienform, Wohnort und -grösse und Einkommenshöhe der Eltern zu nennen (vgl. Messaris 1983; Barthelmes/Sander 1990; Austin 1993; Hurrelmann/Hammer/Stelberg 1996; Livingstone/Bovill 2001; Sander 2001; Kuchenbuch 2003; Warren 2003; Paus-Hasebrink/Bichler 2005, 2006), aber neben objektiven, sozial-ökologisch bestimmbaren Bedingungsfaktoren auch personen- und interaktionsbezogene Prozesse, wie etwa unterschiedlich gelebte familiale Lebensstile. Alle diese Faktoren prägen die Art und Weise, welche Medienangebote Kinder auswählen und präferieren und wie sie diese in ihrem Alltag nutzen (können).

Folgt man dem aktuellen Kinder- und Jugendbericht der Bundesregierung (vgl. Barthelmes 2005) gebührt insbesondere Kindern aus sozial benachteiligten Familien hohe gesellschaftliche Aufmerksamkeit und Verantwortung. Schliesslich führt die Variabilität der Lebensführung der Familien nicht nur zu unterschiedlichen, sondern vor allem auch zu ungleichen Möglichkeiten ihrer Kinder, mediale 
Angebote auszuschöpfen und sich mit ihnen kulturell und sozial in die Gesellschaft einzubringen und an ihr zu partizipieren (vgl. Büchner 2002, S. 492).

Vor diesem Hintergrund widmet sich der vorliegende Beitrag der Frage nach der Relevanz von Kinderfernsehen für Kinder aus sozial benachteiligten Familien; er fragt dabei speziell danach, welche Angebote für diese Kinder Bedeutung gewinnen, welche Programme sie auswählen und wie und mit welchem Ziel sie diese in ihren Alltag einbinden. Ziel des Beitrags ist es, den Blick dafür zu schärfen, dass die Verantwortung dabei sowohl auf Seiten der Produktion und des Angebots, etwa durch speziell auf die Belange und Bedürfnisse von sozial benachteiligten Kindern abgestimmte Programme, als auch auf Seiten der Rezeption durch notwendige medien- und sozialpädagogische Hilfestellungen für Kinder und ihre Familien (vgl. Aufenanger 2004) zu verorten ist.

Zunächst erscheint die Frage klärungsbedürftig, was im Folgenden unter Kinderfernsehen verstanden wird.

\section{Kinderfernsehen: Überlegungen zu einem schwierigen Begriff}

Jeder Diskurs zum Thema Kinderfernsehen ist verbunden mit einer zunehmend schwieriger werdenden Definitionsproblematik des Begriffs: Handelt es sich beim Kinderfernsehen um das Programm, das von speziellen Kinderfernsehredaktionen verschiedener Sendeanstalten für Kinder produziert und verantwortet wird (vgl. Gehrke/Hohlfeld 1997, Biesinger 2004) oder um dasjenige, das Kinder mittlerweile als «ihr» Programm favorisieren (vgl. Gehrke/Hohlfeld 1997), der weiten Begriffsbestimmung von Gert K. Müntefering, dem «Vater» des WDR-Kinderfernsehens, folgend, der die mittlerweile zum geflügelten Wort avancierte Aussage geprägt hat, Kinderfernsehen sei, wenn Kinder fernsehen (vgl. Mundzeck 1991, S. 28)? Oder richtet sich «Kinderfernsehen» nach dem Alter der jungen Rezipienten und Rezipientinnen? Ein solcher Definitionsversuch erscheint zwar recht unpräzise (vgl. Gangloff 1994, S. 163; Biesinger 2004, S. 29), er kann jedoch bereits eine historische Relevanz für sich beanspruchen, zieht man den Begriff des «Vorschulfernsehens» heran, der in den 70er Jahren für das bildungsorientierte, mal mehr kompensatorisch, mal mehr emanzipatorisch ausgerichtete spezielle Programmangebot von ARD und ZDF für Kindergartenkinder geprägt wurde (vgl. Paus-Haase 1986) oder den Beschluss der Ständigen Programmkonferenz der ARD vom 1. November 1958. Dieser trug den wütenden Protesten von Seiten kulturkritisch oder bewahrpädagogisch argumentierender Jugendschützer auf die Ausstrahlung der ersten amerikanischen Serien wie Fury und Texas Rangers Rechnung und "gestattete» keine weitere Ausstrahlung von Sendungen mehr für Kinder unter sechs Jahren (vgl. Gehrke/Hohlfeld 1997, S. 3). Mit diesem Beschluss war dem so genannten «heimlichen Kinderprogramm» Tür und Tor geöffnet. 
Bereits diese wenigen Aspekte umreissen deutlich, dass sich Definitionsversuche, die nur die eine Seite, die der Produzenten und Angebote, bzw. die der anderen Seite, der Rezipienten und Rezipientinnen, also der Kinder selbst, ins Auge fasst, in einem Dilemma stecken bleibt und immer nur - wenn zuweilen auch durchaus pragmatisch - eine Perspektive des Kinderfernsehens beleuchten. Fest steht, dass die Frage nach dem Symbolangebot, das Fernsehprogramme als Bedeutungs- und Sinnfundus in den von Kindern favorisierten Programmen liefern (und dies können speziell für Kinder produzierte Programme, aber ebenso die von ihnen darüber hinaus favorisierten Angebote sein) nicht ohne Blick sowohl auf das Angebot als auch die Rezeption, also darauf, welche Bedeutung Kinder Fernsehangeboten in ihrer Lebenswelt beimessen, angemessen beantwortet werden kann.

Hinzu kommt, quasi als Katalysator, dass sich das Erscheinungsbild des Kinderfernsehens seit der Etablierung der dualen Rundfunkordnung Mitte der 80er Jahre drastisch verändert hat. Schon seit Beginn der 90er Jahre markieren die bei Kindern besonders beliebten Angebote privater Sender, wie seinerzeit RTL Plus, Sat 1 und Tele 5 und heute Super RTL, RTL II oder Pro Sieben einen starken Trend hin zur Kommerzialisierung. Mittlerweile beherrscht der Markt die Entwicklung des Kinderfernsehens. Doch schon 1995 identifizierte Hans-Dieter Erlinger neben Deregulierung, Desintegration und Emotionalisierung die Kommerzialisierung (Erlinger 1995, S. 8) als weiteres zentrales Stichwort in der Entwicklung des Kinderfernsehens; er sah eine besondere Pointe darin, dass gerade in dieser Programmsparte die Marktkriterien am weitesten entwickelt waren: «Nirgendwo sonst gibt es ein Programm, das z. B. bewusst als emotionale Fläche konzipiert ist, wobei sich Zeichentrick, Werbung und Trailer ablösen (z.B. bei RTL oder Sat 1 am Wochenende). Programm wird dabei zum Werbeumfeld» (Erlinger 1995, S. 8).

Heute bestimmen so genannte "Medienmarken»² als crossmedial vermarktete Produkte das Kinderfernsehen und - darüber hinaus - das gesamte Medienangebot für Kinder. Kinderfernsehangebote sind eingebunden in so genannte Produkt- und Markenverbünde (vgl. Dreier/Bichler/Pluschkowitz 2004); Fernsehanbieter wenden auf verschiedenen Angebotsebenen (Programm, Einzelsendung) Markenstrategien an, die sich als «multimediale Strategien der Verwertung von Markenzeichen für Kinder» (ebda) kennzeichnen lassen; ihre erfolgreichen Produkte geniessen als «Medienmarken» marktbestimmende Relevanz. Dabei lassen sich mit Blick auf die angewandten Markenstrategien so genannte «Kinder-» und «Elternmarken» unterscheiden. Während sich die Anbieter bei «Kindermarken» in ihren kommunikativen Massnahmen darauf konzentrieren, vor allem diese Zielgruppe zu erreichen, geht es den Anbietern von Programmen, auf deren Auswahl Erwachsene im Umfeld der Kinder, in den meisten Fällen die Eltern, einen grossen Einfluss haben, darum, diese Zielgruppe mit Informatio- 
nen zu versorgen. Die als Markendreiklang bekannte Formel «Kennen > Mögen $>$ Kaufen» lässt sich in Bezug auf das werbefinanzierte Fernsehprogramm hier in «Kennen > Vertrauen > Auswählen» übertragen, für Pay-TV-Angebote gilt sie in der Variante "Kennen > Vertrauen > Kaufen» (Dreier/Bichler/Pluschkowitz 2004, S. 35). ${ }^{3}$ Die Anbieter von «Kindermarken» richten sich dagegen direkt an die Kinder selbst; Nintendo beispielsweise hat die meisten seiner MarketingMassnahmen zu Pokémon im unmittelbaren Umfeld der Zielgruppe mit Events, Anzeigenkampagnen in Kinderzeitschriften und Werbung im Kinderfernsehen platziert und sich in ihren Produkten auf das Taschengeld-Budget der Kinder ausgerichtet, etwa bei Hörspielauswertungen und Sammelkarten, die in Verbindung mit Fernsehprogrammen erscheinen (vgl. ebda).

Mit Blick auf die Seite der Rezipienten finden sich «Elternmarken» vor allem auf der Senderebene KI.KA und auf der Sendungsebene z. B. in Bob der Baumeister, Löwenzahn, Die Sendung mit der Maus, Petterson und Findus, Logo, Sesamstrasse, Biene Maja und Tigerentenclub. Der Kinderkanal z. B. bietet ein reichhaltiges, weitgehend gewalt- und werbefreies Programm, das Eltern ein gutes Gefühl vermittelt: Auf diesem Sender können die Kinder ihrer Ansicht nach ein Programm finden, das den eigenen Erziehungszielen nicht zuwiderläuft, sie vielmehr sogar unterstützt oder die Eltern zeitweilig von ihren Aufsichts- bzw. Erziehungsverpflichtungen entlastet. "Kindermarken» hingegen stellen multimedial dargebotene Produkte dar, die Kindern die Möglichkeit bieten, ihre Anliegen zu bearbeiten, zumeist deutlich als Abgrenzung von den Eltern und als erste Versuche angelegt, Unabhängigkeit und Eigenständigkeit zu erlangen und zu präsentieren. Kinder nutzen diese Angebote und konstruieren sie als ihre eigene «Medienmarke»; Produkte dieser Art können in Abgrenzung zu den «Elternmarken» als «Kindermarken» bezeichnet werden (vgl. Paus-Hasebrink/Lampert/Hammerer/Pointecker 2004).

Zwar erweist sich auch in diesem Kontext das zuvor beschriebene Dilemma als virulent - schliesslich sind die «Medienmarken» der Produzenten und vice versa ihre «Kinder»- und «Elternmarken» nicht per se deckungsgleich zu setzen mit den «Medienmarken», also den «Kinder»- und «Elternmarken» der Kinder bzw. ihrer Eltern selbst, sprich den Produkten, die den Kindern als Stoff zur Bearbeitung ihrer Anliegen im Alltag oder den Eltern als Garant für kindergeeignetes Programm dienen. Die Studie «Medienkindheit - Markenkindheit» (Paus-Hasebrink/Neumann-Braun/Hasebrink/Aufenanger 2004) lässt aber bei einigen Angeboten des Kinderfernsehens, die den Markt faktisch bestimmen, eine hohe Übereinstimmung erkennen. So treffen etwa Angebote wie Pokémon oder auch Dragonball Z, Yu-Gi-Oh oder Bob, der Baumeister die Interessen der Kinder und - despektierlich formuliert - auch den der Produzenten, denen es mit diesen crossmedial vermarkteten, sinnlich vielfältig erfahrbaren «Überall-Angeboten», zumeist privater Anbieter, gelingt, ihre jungen Rezipienten und Rezipientinnen 
als Kundenklientel zumindest über eine bestimmte Zeitspanne mit Neugier auf die jeweiligen Nachfolgeprodukte an sich zu binden. Mit den Elternmarken KI.KA sowie Sendungen wie Bob der Baumeister, Tigerentenclub und auch Löwenzahn ${ }^{4}$ mit seinen entsprechenden Videos und Computerspielen, die den Erziehungszielen der Eltern nicht entgegenstehen bzw. diese explizit unterstützen, treffen die Produzenten hingegen die Interessen der Eltern.

Dieser Perspektive folgend gilt im Folgenden entsprechend der eingangs formulierten Aufgabenstellung die Aufmerksamkeit den Angeboten des Kinderfernsehens in sozial benachteiligten und anregungsärmeren Milieus. Dazu bezieht sich der Beitrag auf bereits bekannte Studien, aber ebenso auf erste Ergebnisse einer noch nicht abgeschlossenen Untersuchung in Österreich. ${ }^{5}$ Diese geht u. a. den Fragen nach, welche "Medienmarken» des Fernsehens bei Kindern aus sozial benachteiligten Familien und ihren Eltern im Vordergrund stehen, das heisst, welche "Kinderfernsehmarken» sich diese Kinder aus der Angebotsfülle tatsächlich als ihre Marken zu eigen machen, wie diese von den Eltern beurteilt werden und um welche Art von Angeboten es sich dabei handelt, um speziell für Kinder produzierte öffentlich-rechtliche oder private Angebote oder vielmehr um Produktionen für Erwachsene. ${ }^{6}$

\section{Der Fernsehumgang von Kindern aus sozial benachteiligten Familien}

Obwohl die Bedeutung lebensweltlicher Hintergründe und im speziellen sozio-ökonomischer Lebensbedingungen bzw. Voraussetzungen von Kindern und deren Familien aus vielen Studien hervorgeht und inzwischen eine grosse Anzahl an Untersuchungen zum Themenkomplex «Kindheit/Jugend - Medien - Gesellschaft» existiert, lassen sich nur vereinzelt Untersuchungen finden, bei denen der Einfluss sozialer Faktoren auf die Medienrezeption von Kindern (siehe z.B. Kuchenbuch 2003) und in Folge die (Medien-) Sozialisation von Kindern und Jugendlichen (siehe Paus-Hasebrink/Bichler 2006) im Mittelpunkt des Forschungsinteresses steht. Dennoch zeigen sich aus diesen Studien vor allem drei aufeinander einwirkende Bereiche, in denen lebensweltlich-familiale Voraussetzungen besonderen Einfluss auf die Auseinandersetzung von Kindern mit Medien zu zeitigen scheinen: (1) die Medienausstattung der Kinder und ihrer Familien, (2) die Mediennutzungsweise der Kinder und ihrer Familien sowie (3) die elterliche Medienerziehung. Vor allem im Hinblick auf das Lieblings- und Leitmedium der Kinder, den Fernseher, lassen sich hierzu besondere, sozio-ökonomisch und sozio-kulturell bedingte Spezifika des Umgangs und der Auseinandersetzung mit Medien finden.

(1) So wird zwar deutlich, dass Kinder aus sozial besser gestellten Familien in der Regel über mehr Mediengeräte verfügen als Kinder aus sozial benachteiligten Familien. Dabei zeigt sich jedoch, dass dies vorwiegend die «neuen» Medien 
Computer und Internet betrifft, wohingegen das Lieblingsmedium, der Fernseher, sehr viel häufiger in den Kinderzimmern von Mädchen und Jungen aus Unterschichtfamilien zu finden ist und auch bevorzugt dann dort, ohne Aufsicht der Eltern, genutzt wird (vgl. Medienpädagogischer Forschungsverband Südwest 2006, S. 13ff; Jäckel/Wollscheid 2006, S. 586; Paus-Hasebrink/Bichler 2005, S. 106; Süss 2004). Da die Verfügbarkeit eines TV-Geräts im eigenen Zimmer die elterliche Kontrolle des kindlichen Medienumgangs schwächt, verwundert es wenig, dass die durchschnittliche Fernsehnutzungsdauer von Kindern mit eigenen Fernsehapparaten höher ist als jene von Heranwachsenden, die sich im Wohnzimmer ihren favorisierten Sendungen widmen (vgl. Feierabend/Klingler 2007, S. 202). Es lassen sich somit Zusammenhänge zwischen der Fernsehausstattung der Kinder und der Art und Weise ihrer Fernsehnutzung ziehen. Welche Formen der kindlichen Fernsehumgangsformen erscheinen nun bei Heranwachsenden aus sozialen Problemlagen vorherrschend bzw. zentral?

(2) Unabhängig vom sozialen Milieu gilt das Fernsehen nach wie vor als das Lieblingsmedium der Kinder, dem Mädchen und Jungen sich täglich viel und gerne zuwenden (vgl. Feierabend 2006, S 216ff; Feierabend/Mohr 2004, S. 455; Grüninger/Lindemann 2000, S. 106). Doch im Hinblick auf die Nutzungsdauer, die Sendungspräferenzen und den Stellenwert, den Kinder dem Fernseher zuschreiben, lassen sich deutliche Unterschiede zwischen Kindern in unterschiedlichen sozialen Lebenslagen finden. Heranwachsende aus sozial benachteiligten Familien sehen etwa tendenziell länger und häufiger fern als Kinder aus einem sozial gesicherten Elternhaus. Zudem zeigt sich, dass sie dem Fernseher, vielleicht auch in Ermangelung alternativer Freizeitmöglichkeiten (vgl. Roberts/ Foehr/Rideout/Mollyann 1999), einen bedeutend höheren Stellenwert in ihrem Alltag zuschreiben und ihn auch wesentlich unreflektierter nutzen als Kinder aus der Mittel- und Oberschicht (vgl. Jäckel/Wollscheid 2006, S. 586; Fuhs 2002, S. 646; Fuhs 1996, S. 154; Beentjes/Koolstra/Marseille/van der Voort 2001; Süss 2004, S. 150; Grüninger/Lindemann 2000, S. 135; Hurrelmann/Hammer/Stelberg 1996, S. 57). Private Fernsehprogramme zählen dabei zu den Fernsehfavoriten von Heranwachsenden aus sozial schwächeren und anregungsärmeren Milieus (vgl. Kuchenbuch 2003, S. 5ff.; Hurrelmann/Hammer/Stelberg 1996, S. 62f.). Während sich Kinder aus sozial höher gestuften Familien auch häufig informationsorientierte Angebote wie etwa die Sendung mit der Maus oder das öffentlich-rechtliche Kinderprogramm ansehen, fühlen sich ihre Altersgenossen aus sozial schwächeren Familien verstärkt zu den fiktionalen, crossmedial vermarkteten Zeichentrickserien der privaten Sender, beispielsweise zu Animes wie Pokémon oder Dragon Ball (Z), hingezogen (vgl. Kuchenbuch 2003, S. 5ff; PausHasebrink/Lampert/Hammerer/Pointecker 2004, S. 159f.; Bachmair 2005, S. 81). Dies lässt vermuten, dass die Auswahl von «Elternmarken» bzw. "Kindermarken» 
als deklarierte Lieblingsangebote der Kinder u. a. auch von den lebensweltlichen Hintergründen der Mädchen und Jungen geprägt wird: «Elternmarken» werden demnach häufiger von Kindern aus sozial höher gestuften Familien bevorzugt, wohingegen Heranwachsende aus anregungsärmeren Milieus häufiger «Kindermarken» favorisieren. Es wird jedoch deutlich, dass milieuübergreifend vor allem jüngere Kinder (insbesondere im Vorschulalter) in erster Linie Kinderfernsehprogramme im klassischen Sinne, also explizit für sie produzierte Sendungen präferieren (vgl. Medienpädagogischer Forschungsverband Südwest 2006, S. 19; Grüninger/Lindemann 2000, S. 123).

Diese aus vornehmlich allgemein gehaltenen Studien zum Medienumgang und zur Mediensozialisation Heranwachsender ablesbaren Sendungspräferenzen finden sich auch in der oben bereits angesprochenen österreichischen Untersuchung zur Mediensozialisation von Kindern im Vorschulalter aus sozial benachteiligten Milieus bestätigt (vgl. Paus-Hasebrink/Bichler 2006) ${ }^{8}$ :

So werden mehr als die Hälfte der Lieblingssendungen der Mädchen und Jungen auf privatwirtschaftlich finanzierten Fernsehkanälen - vor allem auf Super RTL -, aber auch auf den Premiere-Kindersendern Premiere Junior und Disney Channel sowie vereinzelt auf RTL II ausgestrahlt. Einige der genannten Serien entstammen auch dem öffentlich-rechtlichen Programm ${ }^{9}$ und jene, die nicht dem ausgewiesenen Kinderprogramm zuzurechnen sind und im Regelfall zusammen mit den Eltern rezipiert werden, finden sich zumeist auf RTL. Dabei zeigt sich, dass die Kinder nicht nur aus einem grossen Fundus an Angeboten wählen können, sondern dies auch tun: Es offenbart sich eine grosse Palette an gern und häufig rezipierten TV-Angeboten, da jedes Kind seine ganz speziellen Lieblingssendungen und Sendungspräferenzen hat. Nur wenige bestimmte Sendungen - wie der bei mehr als der Hälfte der Kinder als absoluter Favorit geltende, als «Kindermarke» produzierte und von ihnen auch als solche wahrgenommene Spongebob Schwammkopf oder der Klassiker unter den Kindersendungen Das Sandmännchen - werden von mehreren Kindern als ihre Lieblingsangebote deklariert. ${ }^{10}$ Dabei lassen sich jedoch geschlechtsspezifische Unterschiede bei der Wahl von Lieblingssendungen bzw. -figuren feststellen: So fühlen sich Jungen deutlich zu aktionsreichen Serien mit starken, männlichen Helden wie Herkules, Dragonball, Yu-Gi-Oh, Tom und Jerry, Tarzan oder One Piece, an denen sie deren Kraft und Mut bewundern (als «Jungenmarken» zu bezeichnende Angebote), hingezogen, und Mädchen bevorzugen dagegen stärker alltagsbezogene Serien mit anthromorphen Tierfiguren oder weiblichen Haupthelden wie Simsalabim Sabrina, Charmed, Disneys Gummibärenbande, Bibi Blocksberg, Benjamin Blümchen oder Winnie Puuh.

Crossmedial vermarktete (Zeichentrick-)Sendungen von privaten TV-Anbietern, die im Rahmen der ausgewiesenen Kinderprogramme ausgestrahlt werden und vornehmlich der Klassifikation «Kindermarke» zuzuordnen sind, zählen, so das 
Ergebnis dieser österreichischen Studie, zu den favorisierten Angeboten bei den fünf- bis sechsjährigen Kindern aus sozial benachteiligten Familien.

(3) Zu einem wesentlichen, auf die oben angeführten spezifischen Medien- bzw. Fernsehnutzungsweisen von Kindern aus sozio-ökonomisch und sozio-kulturell benachteiligten Familien Einfluss nehmenden Faktor zählt dabei das wenig regulative und stringente (Medien-)Erziehungsverhalten sowie das eigene Nutzungsverhalten der Eltern, das deutliche Parallelen zum Medienumgang der Kinder aufweist (vgl. Pasquier 2001, S. 170f; Paus-Hasebrink/Bichler 2006; Best 1999; Büchner/Fuhs 1994).

$\mathrm{Da}$ Eltern ihren Kindern durch ihren eigenen Umgang mit dem Fernseher ihre Art des Fernsehgebrauchs vorleben, und da Väter und Mütter aus unterschiedlichen Sozialmilieus und Lebenslagen im Vergleich zu anderen Rezipientengruppen differierende TV-Nutzungsmuster erkennen lassen, ist es wenig verwunderlich, dass sich milieuspezifische Gemeinsamkeiten im Medien- bzw. Fernsehumgang von Eltern und Kindern zeigen (vgl. Kuchenbuch 2003, S. 5ff): Sowohl Kinder als auch Erwachsene aus anregungsärmeren Milieus greifen verstärkt auf das Angebot der kleineren privaten Sender zurück, während sich die Nutzung der grossen Privatsender relativ gleichmässig auf alle sozialen Schichten verteilt. Das Programm öffentlich-rechtlicher Sender stösst hingegen bei sozial besser gestellten Familien auf grossen Anklang.

Hat das Vorleben eines bestimmten Fernsehnutzungsstils durch die Eltern zwar einen bestimmten Imitationseffekt und eine gewisse Vorbildfunktion für die Kinder, so sind für die Ausformung der kindlichen Fernsehumgangsweisen doch vor allem die elterlichen Medienerziehungskonzepte und deren Umsetzung verantwortlich bzw. prägend. Und diesbezüglich zeigen Eltern aus sozial schwachen Milieus häufig grosse Defizite in der Vermittlung eines kompetenten Medienumgangs an ihre Kinder, da in solchen Familien im Regelfall keine transparente, reflektierende und Hilfestellung leistende Medienerziehung vorzufinden ist. So wurde etwa in verschiedenen europäischen Studien (vgl. Pasquier 2001, S. 170f.; Best 1999; Büchner/Fuhs 1994) nachgewiesen, dass Eltern aus sozio-ökonomisch höher gestuften Familien die Mediennutzung ihrer Kinder - vor allem die Zuwendung zu Fernsehangeboten - in einem weitaus höheren Mass kontrollieren als Väter und Mütter aus sozio-ökonomisch ärmeren Verhältnissen. Dabei reglementieren Erstere nicht nur die zeitliche Zuwendung zu Fernsehsendungen, sondern auch die Hinwendung zu bestimmten TV-Angeboten deutlich intensiver und strenger als Eltern in sozialen Problemlagen (vgl. Paus-Hasebrink/ Bichler 2005, S. 106).

Fehlende Kontrolle seitens der Eltern zeitigt an sich nun noch keine negativen Folgen für den Aufbau eines reflektierten und kritischen Medien- und Fernsehgebrauchs ihrer Kinder, sie kann jedoch in unstringenter Handhabung und in 
Kombination mit dem Versäumnis einer kommunikativen, medienkompetenten Schulung (z. B. durch Gespräche über Fernsehserien oder TV-Produktionsmechanismen) sehr wohl dazu führen, dass Kinder nicht adäquat mit dem Medium Fernsehen und mit Medien generell umzugehen wissen. Die bereits angeführte österreichische Studie zur Mediensozialisation von Kindern aus sozial benachteiligten Milieus zeigt in diesem Kontext deutlich, dass Eltern aus sozialen Problemlagen ein sehr inkonsequentes Medienerziehungsverhalten an den Tag legen (vgl. Paus-Hasebrink/Bichler 2006): In jenen Familien, in denen angeführt wird, dass der Medienumgang der Heranwachsenden reglementiert wird, ${ }^{11}$ mangelt es häufig an einer konsequenten Durchsetzung der aufgestellten Regeln und an einer entsprechenden Transparenz dieser Reglementierungen für die Kinder. Und die Eltern, die gar nichts von Medienregeln halten, sprechen von einem bewusst lockeren und ohne (strikte) Regeln erfolgenden Erziehungsstil, den sie jedoch bisweilen unterlaufen, um situativ auf die Fernsehnutzung ihrer Kinder einzuwirken. Ungeeignet erscheinen den Vätern und Müttern dabei in erster Linie Sendungen, die Gewalt beinhalten bzw. zeigen. In der Liste an von den Kindern rezipierten Fernsehangeboten findet sich jedoch eine Vielzahl an Sendungen wieder, die von den Eltern als ungeeignet und verboten deklariert werden (Nachrichten, Real-Life-Formate, Telenovelas, Gewalt beinhaltende Zeichentricksendungen etc.). Die Eltern verurteilen demnach zwar einerseits bestimmte Fernsehangebote als nicht kindgerecht, lassen ihre Kinder jedoch andererseits genau solche Inhalte und Sendungen rezipieren.

Die Unausgewogenheit dieser Form der familialen Medienerziehung zeigt sich auch darin, dass es den zumeist allein erziehenden Müttern und Vätern einerseits wichtig ist, dass ihre Kinder Medien adäquat bedienen und die Funktionsweise von Medien verstehen können, sie es jedoch andererseits im Regelfall an (kommunikativen) Hilfestellungen fehlen lassen. Medienbezogene Gespräche zwischen Eltern und Kindern finden nur sporadisch, zumeist während der seltenen gemeinsamen Fernsehabende, statt. Die Eltern scheinen vielmehr eher wenig Interesse daran zu haben, sich mit dem Fernsehumgang ihrer Söhne und Töchter auseinanderzusetzen. Denn obwohl die Mehrheit der Eltern der Auffassung ist, alle Angebote zu kennen, die ihre Kinder nutzen, lassen sich grosse Diskrepanzen in den Aufzählungen von Eltern und Kindern zu Lieblingssendungen bzw. häufig genutzten Fernsehangeboten der Kinder identifizieren. Dies zeigt, dass die Eltern nicht den Überblick über den Medienumgang ihrer Kinder besitzen, den sie zu haben vorgeben. Bestärkt wird diese Annahme dadurch, dass Kinder sich häufig alleine in ihren Zimmern mit Medien beschäftigen, eine gemeinsame Mediennutzung im Familienverbund kaum stattfindet und medienbezogene Gespräche zwischen Eltern und Kindern Seltenheitswert geniessen (vgl. Paus-Hasebrink/Bichler 2006). ${ }^{12}$ 


\section{Fazit}

Das Fernsehen spielt in sozial benachteiligten Familien eine grosse Rolle; dabei wählen die Kinder, wie erste Ergebnisse einer österreichischen Studie zur Mediensozialisation von Kindern im Vorschulalter aus sozial benachteiligten Milieus zeigen, vor allem crossmedial vermarktete Kinderfernsehangebote privater Sender als ihr Favoritenprogramm aus und machen sich diese Sendungen als ihre «Medienmarken» bzw. "Kindermarken» zu Eigen; sie rezipieren darüber hinaus jedoch auch nicht für sie produzierte Angebote - ebenfalls privater Sender -, die sich an Erwachsene richten, dies jedoch vornehmlich in den (wenigen gemeinsamen) Fernsehabenden mit den Eltern. Von ihren Müttern und Vätern erfahren sie nur wenig Unterstützung, etwa durch eine kommunikative Begleitung ihres täglichen Medienumgangs. Im Gegenteil: Das inkonsequente (Medien-)Erziehungsverhalten der Eltern lässt die Kinder vielmehr mit ihrem täglichen Fernsehkonsum allein. So überlassen häufig die Eltern in sozialen Problemlagen - häufig allein erziehende Mütter - ihre Erziehungsaufgaben den Medien, allen voran dem Fernsehen, da die Mütter und Väter selbst, von ihrer Lebenssituation überfordert, nicht in der Lage sind, ihre Rolle als Wissen und Werte vermittelnde Erziehungsinstanzen zu erfüllen.

\section{Literatur}

Aufenanger, Stefan (2004): Konzeptionelle Überlegungen zu medienpädagogischen Handreichungen für Eltern, Erzieherinnen und Grundschullehrerinnen. In: Paus-Hasebrink, Ingrid/Neumann-Braun, Klaus/Hasebrink, Uwe/Aufenanger, Stefan: Medienkindheit - Markenkindheit. Untersuchungen zur multimedialen Verwertung von Markenzeichen für Kinder. München: Kopaed (Schriftreihe der LPR Hessen, Bd. 18), S. 265-280.

Austin, Erica Weintraub (1993): Exploring the effects of active parental mediation of television content. In: Journal of Broadcasting and the Electronic Media, 2/1993, S. 147-158.

Bachmair, Ben (2005): Qualität des Kinderfernsehens in einer von Alltagsästhetik bestimmten Kultur. In: TelevIZlon, 18/2005/2, S. 78-83.

Barthelmes, Jürgen/Sander, Ekkehard (1990): Familie und Medien. Forschungsergebnisse und kommentierte Auswahlbibliographie. Eine Literaturanalyse zum Medienumgang von Familien. München: DJI-Verlag.

Barthelmes, Jürgen (2005): Zwölfter Kinder- und Jugendbericht: Bildungsorte und Lernwelten. Laufen, Sprechen, Lesen und Reisen - das Entdecken der Welt als Weg zur Bildung. In: DJI Bulletin, Heft 73/2005, S. 20-23.

Beentjes, Johannes W.J./Koolstra, Cees M./Marseille, Nies/van der Voort, Tom H.A. (2001): Children's Use of Different Media: For How Long and Why? In: Livingstone, Sonia/Bovill, Moira (Ed.): Children and Their Changing Media Environment. A European Comparative Study. Mahwah/New Jersey: Lawrence Erlbaum Associates, S. 85-111. 
Begert, Regula/Steinmann, Matthias (1997): Kinder und Medien in der Schweiz. Die Bedeutung der Medien im Alltag der 5- bis 14-jährigen Kinder. Ergebnisse einer bevölkerungs-repräsentativen Umfrage. Bern: Forschungsdienst SRG SSR.

Best, Petra (1999): Medienkompetenz der Kinder verlangt zuallererst Fernseherziehungskompetenz der Eltern. In: Schell, Fred/Stolzenburg, Elke/ Theunert, Helga (Hg.) (1999): Medienkompetenz, Grundlagen und pädagogisches Handeln. München: Kopaed, S. 99-105.

Biesinger, David (2004): Muss Kinderfernsehen gottlos sein? Bedeutung, Chancen und Grenzen des Kinderfernsehens in Deutschland für die religiöse Sozialisation. Münster: LIT.

Büchner, Peter (2002): Kindheit und Familie. In: Krüger, Heinz-Hermann/ Grunert, Cathleen (Hg.): Handbuch Kindheits- und Jugendforschung. Opladen: Leske + Budrich, S. 475-496.

Büchner, Peter/Fuhs, Burkhard (1994): Kinderkulturelle Praxis: Kindliche Handlungskontexte und Aktivitätsprofile im ausserschulischen Lebensalltag. In: du Bois-Reymond, Manuela/Büchner, Peter/Krüger, Heinz-Hermann/Ecarius, Jutta/Fuhs, Burkhard: Kinderleben. Modernisierung von Kindheit im interkulturellen Vergleich. Opladen: Leske + Budrich, S. 63-136.

Dreier, Hardy/Bichler, Michelle/Pluschkowitz, Alois (2004): Multimediale Strategien der Verwertung von Markenzeichen für Kinder. In: Paus-Hasebrink, Ingrid/Neumann-Braun, Klaus/Hasebrink, Uwe/Aufenanger, Stefan: Medienkindheit - Markenkindheit. Untersuchungen zur multimedialen Verwertung von Markenzeichen für Kinder. München: Kopaed (Schriftreihe der LPR Hessen, Bd. 18), S. 28-109.

Erlinger, Hans-Dieter (1995): Geschichte des Kinderfernsehens in der Bundesrepublik Deutschland. Vorstellung eines DFG-Projekts. In: Erlinger, Hans-Dieter (Hg.): Kinderfernsehen II, Siegener Studien Bd. 45. Essen: Verlag Die Blaue Eule, S.157-176.

Feierabend, Sabine (2006): Lebenswelt und Mediennutzung von Vorschulkindern und deren Eltern. In: Frey-Vor, Gerlinde/Schumacher, Gerlinde (Hg.): Kinder und Medien 2003/2004. Eine Studie der ARD/ZDF-Medienkommission. Baden-Baden: Nomos, S. 204-234.

Feierabend, Sabine/Mohr, Inge (2004): Mediennutzung von Klein- und Vorschulkindern. Ergebnisse der ARD/ZDF-Studie "Kinder und Medien 2003». In: Media Perspektiven, 9/2004, S 453-461.

Feierabend, Sabine/Klingler, Walter (2007): Was Kinder sehen. Eine Analyse der Fernsehnutzung Drei- bis 13-Jähriger 2006. In: Media Perspektiven, 4/2007, S. 200-214.

Frey-Vor, Gerlinde/Schumacher, Gerlinde (2004): Kinder und Medien 2003. Studie der ARD/ZDF-Medienkommission. Kernergebnisse für die 6- bis 13-jährigen Kinder und ihre Eltern. In: Media Perspektiven, 9/2004, S. 426-440. Verfügbar über: «http://www.ard-werbung.de/showfile.phtml/ frey-vor_schumacher_9-2004.pdf?foid=12232) (27.11.2006).

Frey-Vor, Gerlinde/Schumacher, Gerlinde (Hg.) (2006b): Kinder und Medien 2003/2004. Eine Studie der ARD/ZDF-Medienkommission. Baden-Baden: Nomos. 
Fuhs, Burkhard (1996): Das ausserschulische Kinderleben in Ost- und Westdeutschland. In: Büchner, Peter/Fuhs, Burkhard/Krüger, Heinz-Hermann (Hg.): Vom Teddybär zum ersten Kuss. Wege aus der Kindheit in Ostund Westdeutschland. Opladen: Leske + Budrich, S. 129-158.

Fuhs, Burkhard (2002): Kindheit, Freizeit, Medien. In: Krüger, Heinz-Hermann/Grunert, Cathleen (Hg.): Handbuch Kindheits- und Jugendforschung. Opladen: Leske + Budrich, S. 637-651.

Gangloff, Tilmann P. (1994): Zwischen Vergnügen und Angst. Was macht das Fernsehen mit den Kindern, was machen Kinder mit dem Fernsehen? In: Gangloff, Tilmann P./Abarbanell, Stephan (Hg.): Liebe, Tod und Lottozahlen. Fernsehen in Deutschland: Wer macht es? Was bringt es? Wie wirkt es? GEP-Buch. Stuttgart: Steinkopf, S. 156-165.

Gehrke, Gernot/Hohlfeld, Ralf (19973): Die duale Rundfunkordnung und das Kinderfernsehen. In: Paus-Haase, Ingrid (Hg.): Neue Helden für die Kleinen. Das (un)heimliche Kinderprogramm des Fernsehens. Münster: LIT, S. 1-34 (1. Aufl. 1991).

Grüninger, Christian/Lindemann, Frank (2000): Vorschulkinder und Medien. Eine Untersuchung zum Medienkonsum von drei- bis sechsjährigen Kindern unter besonderer Berücksichtigung des Fernsehens. Opladen: Leske + Budrich.

Hasebrink, Uwe (2004): Marken als Orientierungspunkte in Mediennutzung und Konsumverhalten von Kindern. In: Paus-Hasebrink, Ingrid/Neumann-Braun, Klaus/Hasebrink, Uwe/Aufenanger, Stefan: Medienkindheit - Markenkindheit. Untersuchungen zur multimedialen Verwertung von Markenzeichen für Kinder. München: Kopaed (Schriftreihe der LPR Hessen, Bd. 18), S. 185-239.

Hradil, Stefan (1987): Sozialstrukturanalyse in einer fortgeschrittenen Gesellschaft. Von Klassen und Schichten zu Lagen und Milieus. Opladen: Leske + Budrich.

Hurrelmann, Bettina/Hammer, Michael/Stelberg, Klaus (1996): Familienmitglied Fernsehen. Fernsehgebrauch und Probleme in verschiedenen Familienformen. Opladen: Leske + Budrich.

Jäckel, Michael/Wollscheid, Sabine (2006): Mediennutzung von Kindern und Jugendlichen in familialen Kontexten. In: Media Perspektiven, $11 / 2006$, S. 585-594.

Kuchenbuch, Katharina (2003): Die Fernsehnutzung von Kindern aus verschiedenen Herkunftsmilieus. In: Media Perspektiven, 1/2003, S. 2-11.

Medienpädagogischer Forschungsverband Südwest (Hg.) (2006): KIM-Studie 2005. Kinder und Medien, Computer und Internet. Basisuntersuchung zum Medienumgang 6- bis 13-Jähriger in Deutschland. Verfügbar über: 〈http://www.mpfs.de/fileadmin/Studien/KIM05.pdf〉 (27.11.2006).

Livingstone, Sonia/Bovill, Moira (Ed.) (2001): Children and their Changing Media Environment. An European Comparative Study. Mahwah, New Jersey: Lawrence Erlbaum Associates.

Messaris, Paul (1983): Family Conversations about Television. In: Journal of Family Issues, 2/1983, S. 293-308.

Mohr, Inge/Schumacher, Gerlinde (2006): Die Rolle der Eltern - Mediennutzung und Erziehungsstile. In: Frey-Vor, Gerlinde/Schumacher, Gerlinde 
(Hg.): Kinder und Medien 2003/2004. Eine Studie der ARD/ZDF-Medienkommission. Baden-Baden: Nomos, S. 33-70.

Mundzeck, Heike (1991): Kinderfernsehen: gestern - heute - morgen. Über Geschichte und künftige Aufgaben des öffentlich-rechtlichen Kinderprogramms. In: Bundeszentrale für politische Bildung (Hg.): Kinderfernsehen - Fernsehkinder. Bonn: Bundeszentrale für Politische Bildung, S. 27-34 .

Pasquier, Dominique (2001): Media at Home: Domestic Interactions and Regulation. In: Livingstone, Sonia/Bovill, Moira (Ed.): Children and Their Changing Media Environment. An European Comparative Study. Mahwah/New Jersey: Lawrence Erlbaum Associates, S. 161-177.

Paus-Haase, Ingrid (1986): Soziales Lernen in der Sendung Sesamstrasse. Versuch einer Standortbestimmung. München: Minerva.

Paus-Haase, Ingrid (1998): Heldenbilder im Fernsehen. Eine Untersuchung zur Symbolik von Fernsehfavoriten. Opladen: Westdeutscher Verlag.

Paus-Hasebrink, Ingrid/Bichler, Michelle (2005): Kindheit im Wandel - Bleiben sozial schwache Kinder auf der Strecke? Ein Plädoyer für die Intensivierung der Forschung zum Medienumgang von Kindern aus anregungsärmeren Milieus. In: TelevIZIon, 18/2005/2, S. 104-107.

Paus-Hasebrink, Ingrid/Bichler, Michelle (2006): Analyse des Wandels von Sozialisation: Veränderung von Kindheit in sozial schwächeren bzw. anregungsärmeren Milieus im Kontext des Wandels der Medien. Zwischenbericht zum Forschungsprojekt Nr. 11136 des Jubiläumsfonds der Österreichischen Nationalbank. Unveröffentlichter Bericht. Salzburg.

Paus-Hasebrink, Ingrid/Neumann-Braun, Klaus/Hasebrink, Uwe/Aufenanger, Stefan (2004): Medienkindheit - Markenkindheit. Untersuchungen zur multimedialen Verwertung von Markenzeichen für Kinder. München: Kopaed (Schriftreihe der LPR Hessen, Bd. 18).

Paus-Hasebrink, Ingrid/Lampert, Claudia/Hammerer, Eva/Pointecker, Marco (2004): Medien, Marken, Merchandising in der Lebenswelt von Kindern. In: Paus-Hasebrink, Ingrid/Neumann-Braun, Klaus/Hasebrink, Uwe/Aufenanger, Stefan: Medienkindheit - Markenkindheit. Untersuchungen zur multimedialen Verwertung von Markenzeichen für Kinder. München: Kopaed (Schriftreihe der LPR Hessen, Bd. 18), S. 135-184.

Paus-Hasebrink, Ingrid /Woelke, Jens /Bichler, Michelle /Pluschkowitz, Alois (2006): Einführung in die Audiovisuelle Kommunikation. München [u. a.]: Oldenbourg Verlag.

Roberts, Donald/Foehr, Ulla/Rideout, Victoria/Mollyann, Brodie (1999): kids \&media@ the new millenium. A Comprehensive National Analysis of Children's Media Use. A Kaiser Family Foundation Report. Verfügbar unter: 〈http://www.kff.org/entmedia/loader.cfm?url=/commonspot/security/getfile.cfm\&PageID=13265; (aufgerufen am 27.11.2006).

Sander, Ekkehard (2001): Common Culture und neues Generationsverhältnis. Die Medienerfahrungen jüngerer Jugendlicher und ihrer Eltern im empirischen Vergleich. München: Leske + Budrich.

Süss, Daniel (2004): Mediensozialisation von Heranwachsenden. Dimensionen - Konstanten - Wandel. Wiesbaden: VS Verlag. 
Warren, Ron (2003): Parental Mediation of Preschool Children's Television Viewing. In: Journal of Broadcasting \& Electronic Media, 3/2003, S. 394 417.

\section{Footnotes}

1 Im Folgenden kann nicht zwischen den unterschiedlichen Klassifizierungen wie Schicht, Status, sozio-ökonomisch reicher oder ärmer ausgestatteten Familien unterschieden werden, da sich die im vorliegenden Beitrag referierten Studien keiner einheitlichen Begrifflichkeit bedienen. Siehe zur Definition sozialer Benachteiligung Fussnote 5 und 6.

2 «Medienmarken» werden als ein symbolisches Beziehungsangebot zwischen den Produkten (eine Marke repräsentiert ein Produkt oder eine Produktgruppe) und den Konsumenten verstanden. Die zentrale Funktion dieser Beziehung ist Orientierung zu bieten, entweder in Bezug auf die Kaufentscheidung selbst oder in Bezug auf den sozialen Wert des Produkts, der eine Positionierung in bestimmten sozialen Kontexten ermöglicht.

3 Dies zeigt sich z. B. daran, dass in den Kinderprogrammen der Pay-TVVeranstalter vergleichsweise alte, den bezahlenden Eltern bekannte Programme wie Die Schlümpfe und Bugs Bunny eine wichtige Rolle spielen (vgl. Dreier/Bichler/Pluschkowitz 2004, S. 35).

4 «Elternmarken» stossen zuweilen auch bei den Kindern selbst auf Interesse; dabei lässt sich ein Zusammenhang zwischen dem (Medien-)Erziehungskonzept der Familien, in denen die Kinder aufwachsen, feststellen (vgl. Paus-Hasebrink/Lampert/Hammerer/Pointecker 2004).

5 Die Studie wird an der Universität Salzburg zunächst über drei Jahre mit Förderung durch den Jubiläumsfonds der Österreichischen Nationalbank von Ingrid Paus-Hasebrink und Michelle Bichler durchgeführt. Sie beschäftigt sich vorrangig mit der Frage, wie sich der allgemeine Medienwandel, auch gerade im Hinblick auf Kommerzialisierungs- und Digitalisierungsprozesse, auf den Prozess der Sozialisation im Hinblick auf einzelne Phasen bei Kindern und Jugendlichen aus sozial benachteiligten bzw. anregungsärmeren Milieus auswirkt.

6 Die Untersuchung folgt in der Bestimmung sozialer Ungleichheit der Definition Hradils. Danach ergeben sich Merkmale sozialer Ungleichheit nicht nur aus klassischen berufsbedingten Dimensionen, sondern diese resultieren aus einem mehrdimensionalen Bedingungsgefüge, bei dem Hradil objektive Dimensionen (Einkommen, Bildung, Geschlecht, Alter, Beruf, Ethnie etc.) und subjektive Dimensionen (Integration, Prestige, sozial begründete Macht) unterscheidet. Zu ihrer Untersuchung schlägt er das Modell der «sozialen Lagen» zur Bestimmung sozialer Benachteiligung vor (Hradil 1987, S. 153). Aus forschungsökonomischen Gründen wurden für die Auswahl der Probanden jedoch ausschliesslich objektive Dimensionen sozialer Ungleichheit herangezogen - in erster Linie die Aspekte «niedriges Einkommen» und «niedriger Bildungsgrad». (Siehe zur Definition der Auswahlfamilien in der "Sozialisationsstudie» ausführlicher: Paus-Hasebrink/Bichler 2005, 2006) Für die Erhebung 
sowie die Auswertung der Studie galt es jedoch auch die subjektiven Ungleichheitsfaktoren mit einzubeziehen. In den hier für den Vergleich der Ergebnisse herangezogenen Studien wurde, abgesehen von einer Untersuchung zur Relevanz von unterschiedlichen Milieus (Sinus-Milieus) für die Mediennutzung von Kindern (Kuchenbuch 2003), die soziale Benachteiligung von Kindern und deren Familien ausschliesslich durch die Merkmale «niedriges Einkommen» und «niedriger Bildungsgrad» definiert.

7 Die qualitative Studie im Kontext der Untersuchung zu «Medienkindheit - Markenkindheit» zeigte, dass Kinder aus Familien in lebensweltlich schwierigen Situationen (Arbeitslosigkeit der Eltern, niedriges Einkommen, Alleinerziehende) sich besonders dem Angebot der kommerziellen Medienwelt zuwenden. Diese Kinder, die sich als Fans von Zeichentrickserien, vor allem fernöstlichen Animes, erweisen, wählen Mediengeschichten und Medienhelden, in denen sie für die Bewältigung ihrer Situation symbolisches Material erhalten (vgl. Paus-Hasebrink/Lampert/ Hammerer/Pointecker 2004).

8 Vgl. Fussnote 4.

9 Diese Sendungen sind vor allem auf KI.KA, dem ORF und den Kinderplattformen der anderen deutschen öffentlich-rechtlichen Fernsehsender zu finden.

${ }^{10}$ Auch andere Studien belegen die individuellen Präferenzen der Kinder bei der Auswahl ihrer Lieblingssendungen. So werden bei einer repräsentativen Befragung zu Medien und ihrer Bedeutung als Marken von 591 Kindern zwischen sechs und dreizehn Jahren 106 verschiedene Lieblingsfiguren genannt, von denen nur wenige eine grössere Zahl an Nennungen erreichen (vgl. Hasebrink 2004, S. 200).

${ }^{11}$ Die Reglementierungen betreffen vor allem das Fernsehen, aber auch die Computer- und Internetnutzung; für die Nutzung der Medien Buch und Radio bedürfen die Kinder nicht dem Einverständnis der Eltern. Verboten werden vor allem jene Medien und Medienangebote, die als ungeeignet für Vorschulkinder erachtet werden (z. B. TV-Sendungen in denen Gewalt gezeigt wird). Oft sind auch zeitliche Reglementierungen hinsichtlich Fernsehnutzung anzutreffen oder es werden «Fernsehverbote» als Sanktionsmittel für ein entsprechendes Fehlverhalten der Kinder eingesetzt (vgl. dahingehend auch ähnliche Ergebnisse aus der aktuellen Studie der ARD/ZDF-Medienkommission von Frey-Vor/Schumacher 2006; 2004, S. 436).

12 Siehe dazu auch: Mohr/Schumacher 2006, S. 62; Feierabend 2006, S. 230; Süss 2004, S. 204; Grüninger/Lindemann 2000, S. 135 ff.; Begert/ Steinmann 1997, S. 71ff.; Hurrelmann/Hammer/Stelberg 1996, S. $108 f .$. 